\title{
Apropiación de habilidades para evaluar información: estudio con alumnos de educación primaria
}

\author{
Guadalupe Vega Díaz * \\ Sylvia Rojas-Drummond \\ Nancy Constantina Mazón Parra. **
}

Artículo recibido:

25 de septiembre de 2007.

Artículo aceptado:

7 de abril de 2008.

\section{RESUMEN}

El artículo presenta los resultados de un estudio sobre los procesos de apropiación de habilidades en alumnos de sexto año de primaria. Para analizar dicho proceso se solicitó a los alumnos que realizaran un proyecto de investigación sobre un tema de su interés que eventualmente sería transformado en una conferencia multimedia. El artículo incluye una breve descripción de la perspectiva sociocultural, la cual enfatiza la importancia de las prácticas sociales y en particular el lenguaje para promover el aprendizaje. Además se retoma el modelo de Wang y Soergel (1998) sobre los elementos y criterios usados para evaluar información.

* El Colegio de México, México. guvega@colmex.mx

** Las últimas dos autoras pertenecen a la Facultad de Psicología de la UNAM, México. (Sylvia: silviar@servidor.unam.mx); (Nancy: nmazón@servidor.unam.mx).

INVESTIGACIÓN BIBLIOTECOLÓGICA, Vol. 22, Núm. 45, mayo/agosto, 2008, México, ISSN: 0187-358X. pp. 79-103 
La metodología aplicada se basa en la etnografía de la comunicación, y se utilizó para analizar las interacciones dialógicas de dos tríadas mientras éstas creaban un texto. Se presentan ejemplos de diálogos que ilustran cómo los alumnos negociaron significados al momento de evaluar la información. También se analizó la toma de decisiones sobre la utilidad de la información para ser incorporada en el texto de su conferencia.

Palabras clave: Alfabetización informacional; $\mathrm{Ha}-$ bilidad para evaluar información; Etnografía de la comunicación; Apropiación de habilidades; Zona de desarrollo próximo.

\section{ABSTRACT}

Appropriation of abilities to assess information: a study with primary students

Guadalupe Vega Diaz; Sylvia Rojas-Drummond and Nancy Constantina Mazón Parra

Results of a study on appropriation abilities in 6th primary graders. The paper describes briefly the sociocultural theory emphasizing the social practices and language to promote learning. The Wang \& Soergel model (1998) is adapted to evaluate information. We present examples of dialogues to illustrate how students negotiated meanings as they were evaluating information, and analyzed student's decision making about the usefulness of information in order to incorporate it to their text for presenting their multimedia conference..

Keywords: Abilities appropriation; Information assessment; Primary education.

\section{INTRODUCCIÓN}

T a UNESCO en su documento Hacia las sociedades del conocimiento, ha en$ـ$ fatizado la importancia de centrar el aprendizaje en el desarrollo de habilidades para ser capaz de "identificar, producir, tratar, transformar, difundir y utilizar la información” de manera crítica y eficiente (UNESCO, 2005, p.29). Estas habilidades han sido englobadas dentro del concepto de Alfabetización 
Informacional (en adelante AI), la cual caracteriza a una persona como aqué1la:

[...]capaz de reconocer cuándo necesita información, que tiene la habilidad para localizarla, evaluarla y usarla efectivamente para satisfacer sus necesidades de información[...] finalmente, una persona alfabetizada en el uso de la información es quien sabe cómo aprender a aprender. Conoce cómo aprender porque conoce cómo se organiza el conocimiento, cómo encontrar información y cómo usarla. Es una persona preparada para el aprendizaje continuo porque puede encontrar la información para contestar preguntas, realizar una tarea o tomar decisiones (ALA, 1989).

En países desarrollados, desde fines del siglo XX la AI es considerada como parte esencial del currículo escolar, de tal forma que existen contenidos integrados a los programas de estudio desde el nivel preescolar hasta la educación superior (Anderson, 1996; Sine, 1994; Utha State Office of Education, 1991; Winsconsin Education Media, 1993;). Los estudios relacionados con la AI se enfocaron inicialmente a analizar los procesos relacionados con el uso de la información partiendo de diferentes modelos y propuestas (Eisenberg, 1990; Kuhlthau, 1989). En los siguientes años se realizaron estudios para evaluar y generalizar los diferentes modelos y propuestas enfatizando los aspectos relacionados con la colaboración entre docentes y bibliotecarios (Berkowitz, 1994; Brock, 1994; Warmkessel y McCade, 1997) y se integraron los programas de AI en el currículo (Todd, 1995). También encontramos estudios dirigidos a analizar los métodos de aprendizaje para la AI (Barrón, 1996; Rekrut, 1997; Tastad, 1997).

En la última década, los estudios han vinculado a la AI con la comprensión lectora y la síntesis de la información (Black, 2005; Eisenberg, 2005; 2005b; Street, 2005). También se reportan estudios que evaluan el aprendizaje de los alumnos (Grimble y Williams, 2004; Nutefall, 2004) y otros dedicados a analizar los procesos de aprendizaje de las habilidades informativas con base en los avances y tendencias en la investigación educativa (Apeji, 2002; Williams y Coles, 2007). Por último se encuentran los trabajos pioneros sobre la creación de comunidades de aprendizaje (Lebbin, 2005).

En el caso de México, un estudio realizado en 1994 (Palacios y Vega) identificó varios programas dirigidos a formar usuarios en el uso de la información, destaca los que entre el propuesto por Dubovoy (1989) denominado "Leer es crecer". Este programa se implementó en escuelas particulares y es uno de los esfuerzos más sistemáticos por introducir contenidos relacionados con el uso de la información en educación primaria. 
En la actualidad parte de las habilidades relacionadas con la AI han sido mencionadas en el Plan Nacional de Desarrollo para el sexenio 2007-2012. Este plan propone como estrategia para elevar la calidad de la educación, que se diseñen programas para que los individuos sean capaces de

[...]analizar, procesar y ampliar la información adecuadamente, de modo que puedan reforzar su aprendizaje, tomar decisiones y abrirse paso en su proyecto de vida. (Poder Ejecutivo Federal [PEF], 2007, p. 410).

Se considera que es urgente desarrollar programas dirigidos a alcanzar la meta mencionada porque se ha encontrado que los alumnos de este nivel tienen serias deficiencias para valorar la información, para comprender lo que leen y para elaborar textos (Instituto Nacional para la Evaluación de la Educación [INEE], 2004; 2005; Secretaría de Educación Pública [SEP], 2007). Esta situación coincide con los resultados reportados por la Organización para la Cooperación y el Desarrollo Económico [OCDE] en sus pruebas PISA (2001; 2003).

En relación con la investigación sobre la AI en educación básica en México, se pueden mencionar algunos trabajos sobre comportamiento informativo de los alumnos de educación primaria (Calva, 2004; 2003) y los relacionados con los hábitos de lectura y su impacto social (Mata, 2007; Ramírez, 2002). También se han identificado trabajos que abordan la AI desde una perspectiva más integral y funcional a partir de la comprensión y la elaboración de textos (Rojas-Drummond y Mercer, 2003; Rojas-Drummond y Peón, 2004; Vega, Mazón y Rojas Drummond, 2007).

No obstante lo anterior se considera que en México hacen falta investigaciones sobre los aspectos relacionados con la AI, principalmente en lo que se refiere a las prácticas educativas, la inserción en el currículo y la relación entre bibliotecarios y maestros. Ello con la finalidad de comprender cómo los alumnos y los profesores perciben, entienden y aplican las habilidades relacionadas con el uso de la información. Es decir, se requieren investigaciones que pasen de la descripción de contenidos y la planeación, al análisis de las personas y de las situaciones en las que se aprende. Acorde con ello, hemos iniciado un estudio que tiene como objetivo encontrar evidencias empíricas sobre cómo los alumnos de sexto año de primaria se apropian de las habilidades relacionadas con la alfabetización informacional. Para realizar dicho estudio nos hemos basado en la perspectiva sociocultural, de la que ofrecemos una breve introducción.

Con este estudio pensamos contribuir a conformar las bases teóricas y empíricas de la AI, y evidenciar la importancia de las investigaciones transdisplinares que en este caso hacen converger a la psicología educativa, la bibliotecología y 
la comunicación. El objetivo final es contar con elementos que nos permitan diseñar actividades de aprendizaje pertinentes y auténticas

\section{iI. Perspectiva sociocultural}

La perspectiva sociocultural pondera el papel del lenguaje y de las prácticas sociales y culturales en la construcción del conocimiento. Uno de los conceptos centrales de esta perspectiva es la zona de desarrollo próximo (ZDP), la cual es definida como:

[...]la distancia entre el nivel real de desarrollo, determinado por la capacidad de resolver independientemente un problema, y el nivel de desarrollo potencial, determinado a través de la resolución de un problema bajo la guía de un adulto o en colaboración con otro compañero más capaz (Vygostsky, 1979, p. 113):

A partir del concepto de ZDP podemos destacar la labor del docente como un soporte cognitivo para que los alumnos resuelvan tareas o problemas que no podrían hacer solos. El alumno por su parte es visto como un ser social que reconstruye el conocimiento en colaboración con otros, con quienes comparte y negocia significados (Rojas-Drummond, Mazón, Fernández y Wegerif, 2006; Rojas-Drummond, y Peón, 2004). En el proceso de construcción del conocimiento el lenguaje es un instrumento mediador que permite la construcción del conocimiento cultural. Por medio del lenguaje los individuos pueden formular sus ideas y comunicarlas, y también pueden pensar y aprender conjuntamente (Mercer, 1997, p.17).

Además del lenguaje, la negociación de significados requiere que los individuos compartan contextos culturales y sociales que les permitan comprender conceptos e ideas, así como realizar acciones en lo que puedan aplicar el conocimiento. Esta forma de concebir el aprendizaje implica la dialéctica constante entre el conocimiento que el alumno ha internalizado, y su contribución para transformar y enriquecer la práctica social y la cultura.

En la perspectiva sociocultural esta internalización es entendida como un proceso de apropiación, es decir un tomar algo que pertenece a otros pero que es transformado por el individuo para convertirlo en propio mediante la realización de actividades culturalmente organizadas; por ejemplo el trabajo y la escuela. Para Leontiev (1993) la apropiación está ligada profundamente a la actividad social y a los instrumentos que son empleados para conocer. Estos instrumentos cumplen una función social que se ha ido conformando de manera histórica (Newman, Griffin y Cole, p. 79-80). 
Una conceptualización más actual del término apropiación la encontramos en Wenger (2001/1998). Su concepto esta referido a la apropiación de significados que serían incorporados a la práctica propia para "[...]utilizar, afectar, controlar, modificar o, en general, afirmar como nuestros los significados que negociamos" (p. 244). Wenger enfatiza que la apropiación no se refiere a objetos o productos, si no más bien que:

1) [...]los significados tienen diversos grados de valor;

2) los participantes pueden tener diversos grados de control sobre los significados que produce una comunidad y, por lo tanto, distintas capacidades para emplearlos y modificarlos;

3) la negociación del significado supone ofertas para hacerse con su propiedad, por lo que la naturaleza social del significado incluye su carácter contestable como una característica intrínseca, (Wenger, 2001/1998, p. 244).

Estos señalamientos nos advierten que los procesos de apropiación no son homogéneos entre personas ni entre comunidades. Y que la comprensión y la aplicación del conocimiento corresponde a esta pluralidad. Pero que no es necesariamente una causa de división, de agresión o de polémica, sino más bien una situación que promueve la creación de otros significados enriquecidos socialmente.

Así en los procesos de apropiación del conocimiento la actividad está mediada por el lenguaje el cual es un instrumento intelectual que permite influir en otros y en nosotros mismos (Wells, 2001, p.116). Para Halliday (citado en Wells, p.122) el lenguaje "[...] es la condición esencial del conocer, es el proceso mediante el cual la experiencia se convierte en conocimiento". Y más aún, permite comprender y reflexionar sobre lo aprendido. Acorde con ello, esta investigación analiza el papel del lenguaje para posibilitar las comprensiones conjuntas y guiar las acciones de los procesos de apropiación de las habilidades informativas; y en particular aquéllas que permiten evaluar la información.

\section{ESQUEMA PARA GUIAR EL PROCESO DE APROPIACIÓN DE LA AI}

Con la intención de identificar las habilidades que se requieren para formar a una persona alfabetizada en información se analizaron los trabajos de Kulhthau (1989, 1990, 1991 y 2005), quien se ha enfocado en el análisis del comportamiento informativo de los alumnos de educación básica estudiando tres aspectos: los sentimientos, los pensamientos y las acciones. También se 
revisó la propuesta de Eisenberg y Berkowitz (1990), quienes proponen el modelo denominado "Las Seis Grandes Habilidades" (The Big6ä), las cuales se vinculan con el aprendizaje basado en la resolución de problemas. Por último, se analizó la propuesta de Stripling y Pitts, (1988) que se enfoca en las habilidades que son necesarias para realizar una investigación.

Tras revisar las propuestas señaladas y la experiencia acumulada en la aplicación de programas de AI en educación primaria durante tres años, se elaboró un esquema para guiar los procesos de apropiación de la AI (Mazón, Rojas-Drummond, Vega y Velez, 2006; Vega, Rojas-Drummond y Mazón, 2007; Vega, RojasDrummond, Rendón y Mazón 2004). Este esquema se representa en la Figura 1.

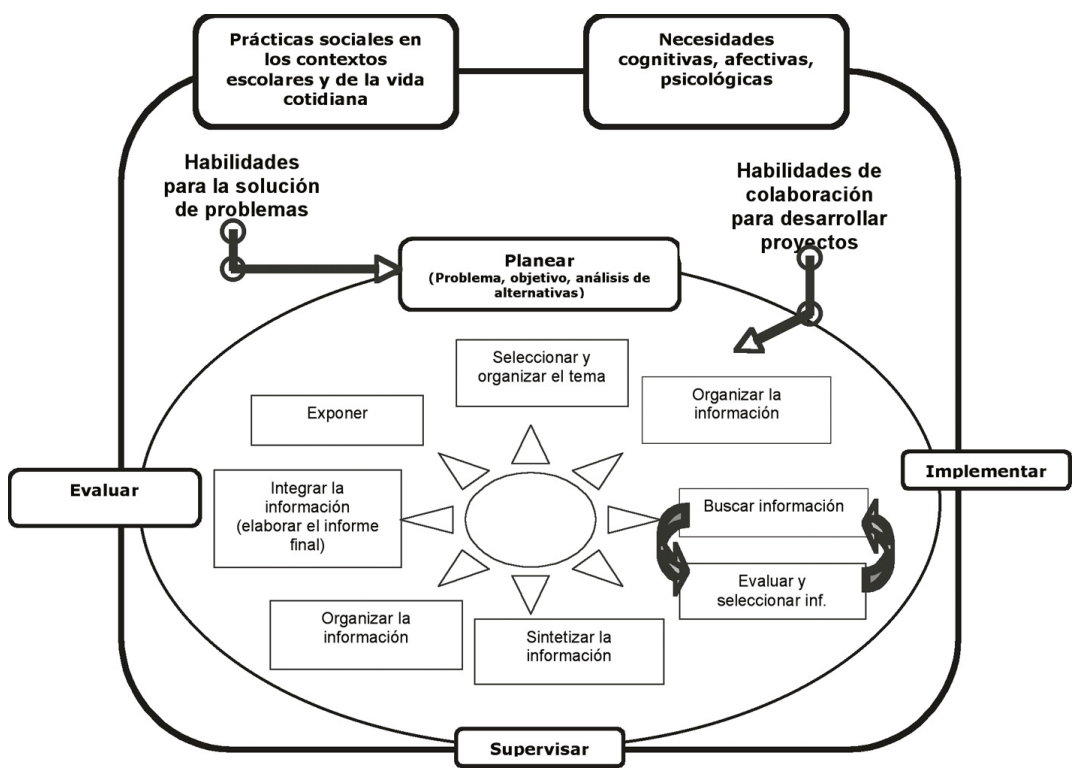

Fig. 1. Esquema para guiar los procesos de Alfabetización Informativa

En el esquema se retoman algunos aspectos de la perspectiva sociocultural. El primero de ellos es el reconocimiento de las prácticas sociales que le dan sentido a las actividades, de las que se derivan las necesidades psicológicas, afectivas y cognitivas de los alumnos (Wilson, 2005: 33). Estos componentes se encuentran ilustrados en la parte superior del esquema.

El siguiente conjunto de habilidades se conforma con habilidades para la solución de problemas. En éste se identifican los siguientes procesos: la planeación compuesta por la delimitación del problema, el objetivo o meta a alcanzar; el análisis de las alternativas, la implementación de estrategias, y la supervisión y evaluación del proceso. 
Otro conjunto de habilidades son las relacionadas con la colaboración, cuyo objetivo es enriquecer las formas de interacción en el aula y fortalecer la comunicación entre los alumnos, para construir el conocimiento. De acuerdo con esto, se promueven una serie de reglas de conversación, basadas en el habla exploratoria (Mercer, 1997).

En la parte central del esquema se encuentran las ocho habilidades informativas. Las cuales son visualizadas como procesos dinámicos e interrelacionados que se aplican de manera estratégica y flexible y no como procesos lineales, estáticos o individuales.

La relación de las habilidades informativas con las prácticas sociales se establece a partir del uso funcional de la oralidad y la escritura. La primera, como ya se mencionó, se promueve en función de la colaboración y la conversación argumentada. La segunda se fortalece en función de la escritura de un texto de tipo expositivo sobre un tema de interés para los alumnos, en el cual expresan necesidades de tipo psicológico, cognitivo o afectivo.

De manera muy breve, los textos expositivos tienen como objetivo comunicar, informar y proporcionarle una explicación al lector acerca de una o varias temáticas (Mazón, 2006, p. 44). Al igual que los textos de tipo narrativo en los que podemos identificar un inicio, un momento cumbre y un desenlace, los textos de tipo expositivo cuentan con estructuras textuales que los hacen comprensibles. Se han identificado cinco tipos básicos de estructuras textuales expositivas: colección, secuencia, comparación-contraste, covariación (también llamada causa-efecto) y problema-solución (Horowitz 1985; Meyer,1984; Sánchez 1993; Stalter y Graves, 1990).

\section{iv. HABILIDAD PARA EVALUAR INFORMACióN}

La capacidad para evaluar información implica el establecimiento y la aplicación de juicios de valor en los cuales inciden los conocimientos previos y el contexto en el que se da la valoración. Como se mencionó arriba, no existen trabajos en México que nos permitan caracterizar las habilidades informativas de los alumnos de educación básica. Debido a ello se ha retomado la caracterización de la American Association of School Librarians [AASL], la cual señala que los alumnos que saben evaluar información son:

[...]capaces de determinar la calidad de la información. Comprenden los principios para valorar la actualidad, la relevancia, la comprensión y la imparcialidad de la información. Los estudiantes aplican estos principios en la selección de fuentes de información en diferentes formatos y usan juicios lógicos e informados para 
aceptar, rechazar o remplazar información de acuerdo con una necesidad en particular (AASL, 1998).

Los indicadores que propone esta Asociación para determinar si una persona sabe o no evaluar la información son:

Indicador 1: Determina la exactitud, la relevancia y el hecho de que la información sea comprensiva.

Indicador 2: Distingue un hecho de un punto de vista u opinión.

Indicador 3: Identifica la información que no es exacta o que es falsa.

Indicador 4: Selecciona la información apropiada para un problema o pregunta.

Wang y Soergel (1998) proponen un modelo que ilustra el proceso para evaluar información. En éste identifican tres fases: la selección, la lectura y la citación. La selección está integrada por la identificación de los elementos descriptivos, y la aplicación de criterios y valores para tomar decisiones (ver Figura 2).

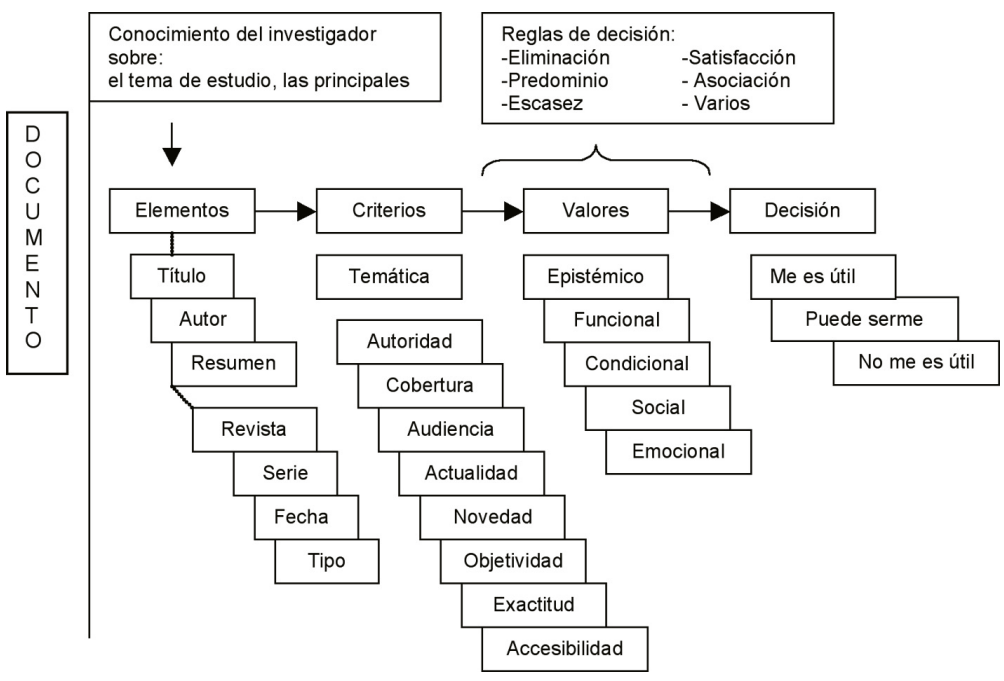

Fig. 2. Modelo para la valoración de documentos (Wang y Soergel, 1998, p. 116)

Los elementos descriptivos se refieren a la identificación del autor, el título, el editor, la editorial y el resumen, entre otros elementos. Por su parte, los criterios de selección se refieren a las cualidades de un documento en función de las necesidades del lector. Algunas de estas cualidades son: 
- Temática. Se refiere al tema de interés de los alumnos, incluyendo las preguntas de investigación y los esquemas.

- Autoridad. Se refiere al prestigio de los autores o editores de un documento.

- Actualidad. Se refiere a las fechas de publicación del documento.

- Idioma. Se refiere al idioma en el que está escrito el documento.

- Formato. Se refiere a los elementos gráficos como imágenes, fotos, sonidos, música, animaciones, etcétera.

- Audiencia. Se refiere al público al que se dirige el documento que se va a escribir, o ante el cuál se expondrán los resultados de la investigación.

Los valores se refieren a los atributos del documento en relación con las necesidades que se intenta cubrir, tales como su valor funcional o la utilidad para una situación específica. El valor epistémico está relacionado con las partes conceptuales del documento. El valor condicional está determinado por las modificaciones que se pueden realizar sobre las necesidades iniciales. El valor social está ligado con el impacto que tiene un tema o autor en una disciplina o área. Por último se encuentra el valor emocional que se vincula con las preferencias o gustos de lector. Tomando en cuenta los elementos, criterios y valores anteriores, el lector toma la decisión sobre si un documento le es útil o no para la necesidad de información que desea cubrir.

En este estudio consideramos que cuando se identifican y aplican los elementos descriptivos y los criterios de selección, se puede determinar la exactitud y la relevancia de la información. También se puede verificar la información falsa de la que no lo es, y por último es posible seleccionar la información que es apropiada para resolver un problema o pregunta.

\section{v. Módulo de AI del Programa Aprendiendo juntos}

Como ya se mencionó la evaluación de la información es una de habilidades del proceso de Alfabetización Informacional. Para este estudio la AI se diseñó como un módulo dentro de un programa más amplio denominado Aprendiendo juntos. Dicho programa pertenece al Laboratorio de Comunicación y Cognición de la Facultad de Psicología de la Universidad Nacional Autónoma de México (Rojas-Drummond, Albarrán, Vega, Zúñiga y Vélez, 2006).

El Programa Aprendiendo juntos tiene aproximadamente seis años de haberse implementando y ha sido un proyecto de la SEP desde el 2004. Su principal objetivo ha sido la creación de comunidades de aprendizaje en algunas escuelas primarias públicas de México. En estas comunidades participan 
de manera activa y creativa alumnos de 3ro a 6to año de primaria, padres de familia, maestros, autoridades e investigadores. En las comunidades se promueve el fortalecimiento funcional de habilidades sociales, cognitivas, psicolingüísticas (lengua oral y escrita) y tecnológicas en alumnos de primaria.

A lo largo de los años de la implementación del programa se han llevado a cabo numerosos estudios longitudinales cuantitativos y cualitativos, en los cuales se han abordado las implicaciones teóricas y prácticas del programa (por ejemplo, Mazón, 2006; Rojas-Drummond y Mercer, 2003; RojasDrummond y Peón, 2004;). El programa promueve diversas habilidades que mantienen una secuencia lógica. Esta secuencia se ajusta de acuerdo con la negociación de los significados y con las actividades específicas que realiza cada grupo de alumnos. En total, el programa dura ocho meses, con sesiones semanales de una hora y media.

Dentro del programa, el modulo de AI se diseñó como un instrumento a través del cual fuera posible observar y analizar los procesos de apropiación de las habilidades informativas de los alumnos de sexto año de primaria. El módulo siguió el esquema para guiar los procesos de AI de la figura 1 .

La primera actividad que realizaron los alumnos fue seleccionar un tema de investigación sobre un aspecto que fuera de su interés. Ello con la finalidad de que los proyectos de los alumnos sean significativos no sólo en el contexto escolar sino, principalmente, en el contexto cultural y social. A partir del tema seleccionado los alumnos elaboraron un esquema para expresar lo que querían investigar y elaboraron sus preguntas de investigación. Con estos elementos iniciaron la búsqueda, evaluación y síntesis de diferentes textos. Y con estos resúmenes prepararon luego la conferencia multimedia. Ésta se presentó oralmente ante una audiencia real amplia al final del ciclo escolar en una Feria cultural. En la audiencia estuvo representada toda la comunidad de aprendizaje.

\section{MÉTOdo}

El estudio tuvo como objetivo encontrar evidencias empíricas de cómo los alumnos de sexto año de primaria se apropian de las habilidades para evaluar la información. Es decir, como comprendían y aplicaban ésta para elaborar resúmenes y para crear la conferencia multimedia.

\section{Participantes}

Los participantes fueron 50 alumnos de sexto año de primaria del ciclo escolar 2004-2005. Se seleccionó este grado escolar para mantener la congruencia 
con los contenidos del programa de estudios de educación primaria, ya que es en este grado en el que se les pide que realicen una investigación escolar apoyada en fuentes de información (SEP, 1993). Los alumnos participantes fueron agrupados en tríadas. En total se formaron dieciséis tríadas y una díada. De las tríadas formadas se seleccionaron dos al azar para darles seguimiento a lo largo del ciclo escolar.

\section{Escenario}

La intervención se realizó en una escuela primaria pública de nivel medio bajo ubicada en el sur del Distrito Federal. La escuela contaba con un salón de medios con 16 computadoras, cañón, biblioteca de aula, maquetas y juegos de mesa, entre otros recursos.

\section{Procedimiento}

La implementación del modulo de AI se efectuó en 30 sesiones que se llevaron a cabo a lo largo de ocho meses. Cada sesión duró 90 minutos, de los cuales los 15 primeros fueron empleados para explicar la actividad, establecer las metas y aclarar los procedimientos para alcanzar éstas. Cuando fue pertinente se realizaron actividades para que los alumnos recordaran lo visto en sesiones pasadas. Acto seguido los alumnos trabajaban en tríadas para buscar, evaluar y sintetizar información sobre su tema de investigación; a esta actividad se le destinó aproximadamente 60 minutos por sesión. Los minutos restantes eran empleados para realizar una reflexión metacognitiva que consistía en vincular lo aprendido en clase con otras actividades escolares y con la vida cotidiana. En ese tiempo los alumnos también compartían sus experiencias y presentaban sus dudas y los avances de su investigación ante sus compañeros.

Las triadas seleccionadas para el estudio estuvieron integradas por dos niñas y un niño. Las dos tríadas realizaron sus actividades en el salón de medios de la escuela. En el escenario se encontraban otros alumnos trabajando, su maestro y el facilitador. Los alumnos tenían la libertad de consultar a cualquiera de los participantes para solucionar sus dudas o resolver problemas, también podían levantarse a consultar información impresa, en discos compactos o en Internet.

Para estudiar los procesos de apropiación se videograbaron las interacciones dialógicas de las dos tríadas cuando estaban realizando sus proyectos de investigación. En este estudio se analizan dos de las cuatro sesiones en las que los alumnos estuvieron realizando resúmenes sobre su investigación, 
para lo cual necesitaban evaluar la información. El método que se aplicó para analizar las interacciones dialógicas fue el de la etnografía de la comunicación (Hymes, 1972), metodología se compone de tres elementos relacionados jerárquicamente:

1) la situación comunicativa, esto es, el contexto y situación específica en el que se da la comunicación;

2) el evento comunicativo, el cual está determinado por cada uno de los propósitos específicos que completan una tarea; por ejemplo en el caso de la elaboración del texto expositivo, están la selección del tema, la organización de éste y, la búsqueda de información, entre otros;

3) los actos comunicativos, los cuales se refieren a las interacciones dialógicas específicas, por ejemplo, hacer una broma o presentar un argumento.

Cabe señalar que ésta metodología ha sido aplicada para investigar la conversación en el aula en México (Mazón, 2006; Rojas-Drummond, Mazón, Fernández y Wegerif, 2006). El proceso de análisis que se llevó a cabo fue el siguiente:

- Transcripción de los diálogos correspondientes a las sesiones de evaluación de la información.

- Identificación de la situación comunicativa en relación con la actividad que se realizó.

- Identificación y análisis de los eventos comunicativos.

- Clasificación de los diálogos de acuerdo con los eventos comunicativos.

- Identificación y análisis de los actos comunicativos en relación con los criterios de evaluación de Wang y Soergel (1998):

A continuación se presentan los datos que se obtuvieron a través del procedimiento mencionado anteriormente.

\section{viI. Resultados}

En términos generales se observó que la comunicación entre los alumnos se desarrolló de manera dinámica. Es decir, podían realizar dos procesos de manera simultánea (por ejemplo, buscar y evaluar información al mismo tiempo) o podían dejar una tarea pendiente y luego retomarla. En ocasiones 
los alumnos dialogaron sobre temas que no estaban relacionados directamente con la tarea asignada; diálogos que no fueron incluidos en el análisis. A continuación se describen la situación comunicativa, los tipos de eventos y las categorías de actos que se encontraron en cada tríada.

Situación comunicativa de la Tríada 1: Esta tríada seleccionó el tema "Las causas del fin del mundo". Las estructuras textuales que aplicaron fueron las de causa-efecto y colección. Sus preguntas de investigación fueron: ¿Qué causas provocarán el fin del mundo? ¿Cómo podemos evitarlo? ¿Qué pasará ese día? A sugerencia del facilitador, los alumnos incluyeron la pregunta ¿Cuáles causas son ciertas y cuáles son falsas? Los alumnos realizaron las actividades y tomaron decisiones de manera conjunta durante todo el proceso.

Las interacciones dialógicas de las dos sesiones analizadas fueron clasificadas en siete tipos de eventos comunicativos y catorce categorías de actos comunicativos diferentes. Dada la naturaleza de la situación comunicativa, la mayoría de los actos identificados estuvieron relacionados con la evaluación de la información (ver Tabla 1). En consecuencia, los resultados reportaron que en siete de los catorce eventos los alumnos aplicaron uno o varios criterios para valorar la información. En total fueron cincuenta y un turnos en los que se hizo referencia al criterio de "Temática", en seis al de "Autoridad", en cuatro al de "Formato", en uno al de "Actualidad", y en uno al de "Audiencia".

Tabla 1. Tipos de eventos y actos comunicativos de la tríada 2

\begin{tabular}{|c|c|c|}
\hline $\begin{array}{l}\text { Tipos de } \\
\text { Eventos } \\
\text { comunicativos }\end{array}$ & Categorías de Actos comunicativos & $\begin{array}{l}\text { Frecuencia } \\
\text { de actos } \\
\text { comunicativos }\end{array}$ \\
\hline Planeación & $\begin{array}{l}\text { 1. Aplicación del criterio de temática para la definición de la tarea } \\
\text { 2. Organización de la tarea }\end{array}$ & $\begin{array}{c}13 \\
7\end{array}$ \\
\hline $\begin{array}{l}\text { Búsqueda de } \\
\text { información }\end{array}$ & $\begin{array}{l}\text { 3. Identificación de recursos de información } \\
\text { 4. Aplicación de los criterios de temática, autoridad, confiabilidad } \\
\text { y actualidad para la determinación de las palabras o frases de } \\
\text { búsqueda } \\
\text { 5. Estrategia de búsqueda }\end{array}$ & $\begin{array}{l}3 \\
5 \\
5\end{array}$ \\
\hline Lectura del texto & $\begin{array}{l}\text { 6. Lectura exploratoria. Aplicación de los criterios de temática, } \\
\text { autoridad y confiabilidad para la revisión de un texto } \\
\text { 7. Lectura profunda. Aplicación de los criterios de temática y de } \\
\text { autoridad en la lectura detallada de un texto y en la selección de las } \\
\text { partes del mismo. }\end{array}$ & 10 \\
\hline $\begin{array}{l}\text { Elaboración } \\
\text { y revisión del } \\
\text { resumen }\end{array}$ & $\begin{array}{l}\text { 8. Aplicación de los criterios de temática y de autoridad para } \\
\text { elaborar el primer borrador completo del texto } \\
\text { 9. Elaboración de la ficha bibliográfica. } \\
\text { 10. Revisión por parte del facilitador. }\end{array}$ & $\begin{array}{c}2 \\
3 \\
3 \\
11\end{array}$ \\
\hline $\begin{array}{l}\text { Corrección del } \\
\text { resumen }\end{array}$ & $\begin{array}{l}\text { 11. Aplicación de los criterios de temática y confiabilidad para la } \\
\text { lectura de fragmentos del texto } \\
\text { 12. Integración del texto }\end{array}$ & $\begin{array}{l}2 \\
2\end{array}$ \\
\hline
\end{tabular}




\begin{tabular}{|c|c|c|}
\hline $\begin{array}{l}\text { Revisión final y } \\
\text { conclusión }\end{array}$ & $\begin{array}{l}\text { 13. Aplicación del criterio de temática en la revisión por parte del } \\
\text { facilitador } \\
\text { 14 Conclusión }\end{array}$ & 5 \\
\hline $\begin{array}{c}\text { Total de } \\
\text { eventos:8 }\end{array}$ & $\begin{array}{r}\text { Total de actos: 14 } \\
\text { Total de tipos de actos en los que aplicaron los criterios para } \\
\text { evaluar la información: } 7\end{array}$ & 86 \\
\hline
\end{tabular}

* Las itálicas tienen como propósito enfatizar los actos en los que se aplicaron los criterios para valorar la información

A continuación se presentan dos ejemplos de los diálogos que sostuvieron los alumnos de la tríada 1 en los que se ilustran la forma en la que aplicaron los criterios de "Temática" y "Audiencia" para evaluar de información (Ejemplo 1).

Ejemplo 1. Tríada 1

Evento comunicativo: Elaboración y revisión del resumen. Los alumnos están realizando un mapa mental a partir de la lectura de un texto de Internet. La pregunta de investigación que están respondiendo es ¿Cuáles causas son reales y cuáles son falsas?

1Rebeca: A ver yo te lo dicto... rayos gamma.. Asteroides ...

20mar: Pero ¿no es lo que ya hicimos?

3Camelia: Si pero hay que completarlo...

40mar: ¿Cuántos son reales?

5Camelia: A ver... rayos gamma... que más... agujeros negros... asteroides

60mar: Guerra nuclear...

7Camelia: No se...

80mar: Nada más plagas mundiales, calentamiento terrestre, guerra mundial, robots asesinos, ya.

9Camelia: ¿Robots asesinos?

100mar: Robots asesinos que toman el poder.

11Camelia: ¿Qué más dice? [al momento de leer el texto va preguntando] ¿lo de los agujeros negros puede ser real?

120mar: No es que no... que se coman a la tierra... no

13Camelia: ¿El impacto de un asteroide?

140mar: Si.

15Camelia: ¿El calentamiento terrestre?

160mar: Si claro.

17Camelia: Gracias por tu cooperación... he aprendido algo ¿ Fb el sol se convierte en una estrellita?.

180mar: No.

19Camelia: ¿No?... entonces son cuatro

En la conversación se observa cómo los alumnos dialogan sobre la naturaleza de la tarea que van a realizar (turnos 1-2). A partir de los turnos 4 al 19 se observa cómo los alumnos aplican el criterio de "Temática" para comprender y seleccionar información para responder una de las preguntas de su proyecto de investigación. Al mismo tiempo realizan la comprensión conjunta del texto y van tomando decisiones sobre si la información que leen es pertinente. En el turno 19 llegan a una conclusión. 
En la actividad Rebeca no interviene en los diálogos pero permanece atenta. Camelia le pregunta a Omar para evaluar el texto conforme va leyendo. Omar va razonando cada una de las causas, pero no hay un cuestionamiento profundo por parte de Camelia, quien se conforma con las respuestas del primero. Por su parte Omar no verbaliza las justificaciones (ver por ejemplo los turnos 5 a 7), en donde las respuestas son dicotómicas.

El ejemplo 2 muestra una interacción diferente, en ella interviene el facilitador realizando preguntas para que los alumnos verbalicen los razonamientos, con los cuales fueron evaluando el texto.

Ejemplo 2. Tríada 1

Evento comunicativo: Revisión del texto elaborado por los alumnos.

20 Facilitador: Pero miren por ejemplo aquí [señala el texto]... ¿Estas son las causas reales?.

21 Omar: Si.

22 Facilitador: ¿Todas son posibles? ¿El impacto de un asteroide?

23 Omar: Si... porque si pasa un asteroide demasiado cerca, nos pasa como a los dinosaurios.

24 Facilitador: Bien, como el [asteroide] de Yucatán.

25 Omar: Como el que se estrelló en la Península de Yucatán

26 Facilitador: [Lee otra oración] Explosiones de rayos gamma ¿Qué significa?

27 Omar: Lo dice acá

28 Facilitador: Ah... pero ustedes no dicen en su documento que significan... si un papá les pregunta que

es un rayo gamma, que van a decir... Entonces ¿cómo pondríamos lo de los rayos gamma?

Imagínense una explosión 10 veces mayor a la del sol [lee en voz alta el texto]... ¿Entonces qué son los

rayos gamma.

29 0mar: Son rayos diez veces más poderosos que los del sol que la luz solar

30 Rebeca: Pero también quema

31 Omar: Explotaría la atmósfera.

En el ejemplo se observa la introducción del criterio de "Audiencia" para decidir la información que les falta integrar (turno 28). El rol del facilitador es ayudar a los alumnos a comprender el texto pensando en la audiencia, para ello les recuerda la pregunta que están tratando de contestar. Lee en voz alta parte del texto que los alumnos han elaborado y va revisando con ellos cada uno de los párrafos (turnos 20-28). También hace evidente que no han completado las ideas y que les falta leer con más detenimiento el documento que seleccionaron. En estos diálogos las justificaciones de los alumnos son más explícitas y amplias.

Situación comunicativa de la Tríada 2. La tríada 2 seleccionó el tema de las "Focas de Ártico". Las estructuras textuales que usaron son: definición, colección y problema-solución. Las preguntas de investigación que se plantearon en su investigación fueron: ¿Qué es una foca? ¿Cuántos tipos de focas hay? ¿Cuánto tiempo tardan en nacer? ¿Por qué las matan? En esta tríada los alumnos se repartieron algunas de las actividades y otras las realizaron juntos. 
En total se identificaron 7 tipos de eventos comunicativos y 12 categorías de actos comunicativos que corresponden a turnos (ver tabla 2).

Tabla 2. Eventos y actos comunicativos de la tríada 2

\begin{tabular}{|c|c|c|}
\hline $\begin{array}{l}\text { Tipos de Eventos } \\
\text { comunicativos }\end{array}$ & $\begin{array}{l}\text { Ubicar en cada categoría los criterios de evolución } \\
\text { equipáralos como Actos comunicativos }\end{array}$ & $\begin{array}{l}\text { Frecuencia } \\
\text { de actos } \\
\text { comunicativos }\end{array}$ \\
\hline Planeación & $\begin{array}{l}\text { 1. Aplicación del criterio de temática para la definición de la } \\
\text { tarea. } \\
\text { 2. Organización de la tarea }\end{array}$ & $\begin{array}{l}9 \\
3\end{array}$ \\
\hline $\begin{array}{l}\text { Búsqueda de } \\
\text { información }\end{array}$ & $\begin{array}{l}\text { 1. Identificación de recursos de información } \\
\text { 2. Aplicación de los criterios de temática, autoridad, } \\
\text { confiabilidad y actualidad para la determinación de las } \\
\text { palabras o frases de búsqueda } \\
\text { 3. Estrategia de búsqueda }\end{array}$ & $\begin{array}{l}6 \\
6 \\
3\end{array}$ \\
\hline Lectura del texto & $\begin{array}{l}\text { 4. Aplicación de los criterios de temática, autoridad y } \\
\text { confiabilidad para la revisión de varios textos } \\
\text { 5. Aplicación de los criterios de temática y confiabilidad en la } \\
\text { lectura detallada del texto (grupal) }\end{array}$ & 4 \\
\hline $\begin{array}{l}\text { Elaboración del } \\
\text { texto }\end{array}$ & $\begin{array}{l}\text { 6. Redacción del texto } \\
\text { 7. Aplicación de los criterios de temática y autoridad en la } \\
\text { revisión del texto. }\end{array}$ & 3 \\
\hline $\begin{array}{l}\text { Lectura selectiva } \\
\text { y corrección del } \\
\text { texto }\end{array}$ & $\begin{array}{l}\text { 8. Aplicación de los criterios de temática y confiabilidad para la } \\
\text { lectura y selección de fragmentos del texto }\end{array}$ & 1 \\
\hline $\begin{array}{l}\text { Revisión, } \\
\text { corrección y } \\
\text { conclusión }\end{array}$ & $\begin{array}{l}\text { 9. Aplicación del criterio de temática en la revisión por parte del } \\
\text { facilitador } \\
\text { 10. Conclusión }\end{array}$ & $\begin{array}{l}2 \\
1\end{array}$ \\
\hline Total de eventos:7 & $\begin{array}{c}\text { Total de actos: } 12 \\
\begin{array}{c}\text { Total de tipos de actos en los que aplicaron los criterios para } \\
\text { evaluar la información: } 7\end{array}\end{array}$ & 41 \\
\hline
\end{tabular}

* Las itálicas tienen como propósito enfatizar los actos en los que se aplicaron los criterios para valorar la información

De los 41 actos comunicativos, 21 corresponden a la comprensión o aplicación de los criterios de valoración, lo que representa un 66\%. De éstos 27 actos, 20 se refirieron al criterio de "Temática", tres al de "Autoridad"; tres actos correspondieron al criterio de "Formato" y uno al de "Idioma". A continuación, se muestran dos ejemplos de diálogo en donde los alumnos aplicaron los criterios de valoración. 
Ejemplo 3. Tríada 2

Evento comunicativo: Busqueda de información. Los alumnos han realizado una búsqueda de información en Internet y han recuperado una serie de documentos que están evaluando juntos.

1Facilitador: ¿Qué están buscando?

2Carla: Las focas del Ártico.

3Manuel: Ah... yo también voy a buscar eso.

4Carla: ¡Noo0; pon otra cosa.

5Teresa: Vamos a poner biología.

6Carla: Mejor zoológico.

7Teresa: ¿Zoología? [Carla escribe "zoología" y realiza la búsqueda].

8Edgar: Se acabo muy rápido [Se oye la campana para salir a descanso].

9Manuel: ¿Me prestas la revista?

10Felipe: Si... ¿Qué dice? [se refiere al texto de Internet]

11Teresa: [Lee el texto en voz alta] Página que trata todo sobre la biología de los mamíferos. Esta nos

puede servir ... pero tiene punto com.

12Edgar: ¿Por qué no nos podemos meter en punto com? [se dirige al facilitador]

13Facilitador: Porque luego sale información que no es buena para niños de tu edad.

14Edgar: Cuando voy al trabajo de mi papá, me mete a unas páginas que tienen punto com.

15Facilitador: Bueno... si... es verdad, lo que hay que hacer es leer el resumen... así vemos si es buena para niños, como lo hicimos ahora.

En el diálogo anterior podemos observar dos aspectos de la evaluación de la información. El primero se refiere a la ubicación del tema en función del área (turnos 1-7). El segundo aspecto tiene que ver con las experiencias previas de los alumnos en el uso de Internet, las cuales les permiten formar sus propios criterios de valoración (turnos 11-15). En el diálogo, uno de los alumnos cuestiona al facilitador sobre las paginas web comerciales (turno 12). El facilitador incorpora lo dicho por E y modifica la aplicación del criterio (turno 15). Al mismo tiempo los alumnos validan sus criterios, los cuales aplicarán posteriormente en la búsqueda de información.

Ejemplo 4. Tríada 2

Evento comunicativo: Busqueda de información. Los alumnos van a buscar información en Internet. F. les ayuda a buscar y a evaluar el documento.

16Facilitador: Les falta ¿cuántos tipos de focas hay? Ahorita vamos a responder eso ¿Cuántos tipos de focas hay? Para eso vamos a buscar en Internet.

17Teresa: Pero ya es una pregunta muy específica. .

18Facilitador: Vamos a escribir exactamente lo que queremos saber

19Teresa: Cuántos tipos de focas hay.

20Facilitador: Vamos a poner que sólo páginas en español. Entonces le ponemos aquí. Ahora hay que tener cuidado con las terminaciones... hummm esta es punto com... esta punto es, esta es española. Vamos a

seguir... tipos de... focas ... vamos a entrar a este.

21Teresa: ¿Este?

22Facilitador: El que dice tipos de focas...las focas pertenecen a la...foca costera.

23Teresa: No.

24Facilitador: ¿Este?...¿Qué estamos buscando? 


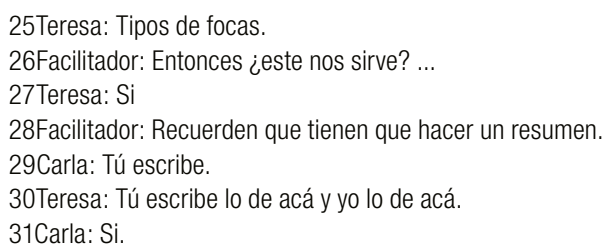

En el ejemplo 2, los alumnos están buscando información en Internet utilizando frases sobre su tema de investigación (turno 19). Los criterios de valoración qué aplican son los de "Temática" (turnos 22-25) y el de "Autoridad" (parte del turno 20). Además de ello, los alumnos limitan su búsqueda por idioma (parte del turno 20). Otro aspecto a resaltar es el diálogo sobre la forma en la que los alumnos se distribuyen la escritura del texto (turnos 29-31). Esta forma de trabajo incidió de manera importante en la cantidad de diálogos que los alumnos sostuvieron, sin embargo no afectó el logro de la meta.

En ambas tríadas el criterio de temática fue el que estuvo presente en más actos comunicativos. Esto resulta importante porque evidencia que los alumnos mantuvieron el interés en su tema, y que orientaron la búsqueda de información de manera adecuada a partir de las preguntas y esquemas de investigación.

\section{Discusión}

A partir de los resultados obtenidos podemos afirmar que para los propósitos de la tarea solicitada, los alumnos comprendieron la habilidad para evaluar información y la aplicaron estratégicamente para elaborar sus resúmenes. En este proceso los alumnos que participaron en el estudio desarrollaron su propia forma de colaborar. En la tríada 1 los alumnos realizaron las actividades y aplicaron los criterios de selección de manera conjunta. En contraste, en la tríada 2 se repartieron algunas de las actividades tales como la lectura y la escritura de los resúmenes. En ambos casos el lenguaje fue un instrumento importante para comprender la tarea y para expresar sus dudas, y también para recibir y dar apoyo cognitivo, principalmente entre ellos mismos.

En ambas tríadas se observó que los alumnos sostuvieron diálogos sobre la comprensión o la aplicación de los criterios de valoración. En la tríada 1 un $73 \%$ de los diálogos fueron para evaluar información, mientras que la tríada 2 representó un $66 \%$ de los diálogos. Este dato refleja que la evaluación requirió de comprensiones conjuntas y de la negociación de significados en 
relación con los elementos y criterios para seleccionar información, lo que se puede observar en los ejemplos incluidos en este artículo. También podemos observar que las comprensiones conjuntas se derivaron de una actividad que les resultó significativa y motivante. $\mathrm{Al}$ respecto cabe señalar, que muy pocos diálogos correspondieron a conversaciones sobre aspectos que no estaban ligados con la tarea.

En el análisis de los diálogos fue posible observar que la evaluación de la información implicó diferentes formas de lectura. A estas formas las hemos denominado lectura exploratoria, lectura profunda y lectura selectiva. La primera se dio cuando los alumnos revisaron los listados o referencias bibliográficas que resultaron de sus búsquedas de información. A partir de esta lectura los alumnos seleccionaron un texto fijándose en elementos como el título, los primeros párrafos o las imágenes.

Una vez seleccionado el texto, los alumnos lo leyeron con mayor profundidad y realizaron el resumen solicitado. La tercera forma de lectura se dio después de la revisión del texto por parte de ellos o del facilitador. Esta lectura fue más selectiva y se enfocó a los aspectos concretos que había que completar o corroborar. Es importante señalar que se requiere profundizar más en el análisis sobre los tipos de lectura, a fin de relacionarlos con las propuestas de comprensión lectora (PISA, 2003, 2001; INEE, 2004).

Con respecto a los indicadores propuestos por la AASL, se considera que de manera general estos indicadores fueron observables a través de los diálogos entre los alumnos. Por ejemplo, los alumnos sostuvieron conversaciones sobre la relevancia de la información en función del tema de investigación. También conversaron si la información que recuperaban era apropiada para responder las preguntas de investigación que se habían planteado.

En relación con la metodología de Hymes (1972), ésta resultó de gran utilidad porque permitió observar la dinámica del proceso de evaluación de la información. Dicha dinámica se caracterizó por ser estratégica y flexible, y también permitió sistematizar y clasificar los diálogos de los alumnos. Esta clasificación, sin ser concluyente, nos permitió observar que la habilidad para evaluar información se conforma de una serie de procesos que estuvieron ligados a las diferentes formas de lectura identificadas. Así, las estrategias didácticas para aprender a valorar la información deberían estar ligadas a las formas de lectura, además de los procesos y metodologías de investigación.

Para finalizar esta parte del estudio queremos resaltar la importancia de diseñar estrategias de aprendizaje basadas en entornos en los que se promueva la comprensión y la resolución de problemas en colaboración. También es preciso enfatizar el diseño y la aplicación de estrategias de aprendizaje que incluyan actividades auténticas que estén insertadas en ambientes en los que 
los alumnos puedan discutir, intercambias ideas y mantener la interdependencia positiva con sus pares y sus maestros. Estas actividades son necesarias para que los alumnos se responsabilicen de su aprendizaje y se sientan motivados a utilizar los recursos de información de manera funcional a lo largo de toda su vida, tanto en la escuela como en la vida diaria.

\section{BiBLIOGRAFÍA}

Apeji, A. (2002), "The role of the school library in promoting a reading culture", en Education Libraries Journal, 45 (3) : 27-30.

American Association of School Librarians (1998), Information literacy standards for student learning, Washington, D.C., American Library Association.

American Library Association (1989), Presidential Committee on Information Literacy, (ALA, 1989), Final report, Chicago, Ill., American Library Association.

Barrón, D. (1996), Constructivism : new ways to love them to learn, en School Library media activities monthly, 12(6): 48-50.

Berkowitz, R. (1994), "Collaboration : partnerships for the instructional improvement", en School library media activities montbly, 10(79), 32-35.

Black, S. (2005), "Teaching students to think critically", en Education digest : essential readings condensed for quick review, 70(6):42-47.

Brock, K. (1994), "Developing information literacy through the information intermediary process : a model for teacher-librarians and others", en Emergency Librarian, 22(1), 16-20.

Calva, J. (2004), "La investigación sobre las necesidades de información en comunidades de usuarios”, en Investigación Bibliotecológica: archivonomía, bibliotecología e información, México, D.F., Centro Universitario de Investigaciones Bibliotecológicas, 18(37). , (2003), "El usuario de la información y la biblioteca escolar", en XXXIV Jornadas Mexicanas de Biblioteconomía. México, D.F., AMBAC.

Cortés, J., González, D., Lau, J., Moya, A.L., Quijano, A. Rovalo, L. y Souto S., comps. (2004), "Normas para la alfabetización informativa en educación superior: declaratoria Tercer Encuentro sobre Desarrollo de Habilidades Informativas", en J. Lau y J. Cortés, (eds.), Normas de alfabetización informativa para el aprendizaje, Ciudad Juárez, Chihuahua, México.

Duboboy, S. (1989), Leer es crecer. México: Consejo Nacional para la Cultura y las Artes, 9 vols.

Einsenberg, M. y R. Berkowitz (1990), Information problem-solving: the big six skills approach to library and information skills instruction, Norwood, NJ., Ablex. 
Eisenberg, M. (2005), "Synthesis-where it all comes together", en $\mathrm{Li}$ brary media connection, 24(2), 26.

, (2005b), "Use of information : getting to the heart of the matter", en Library media connection, 24(1), 30.

Horowitz, R. (1985), “Patterns of texts: part 1", en Journal of reading, 28(5), 448-454.

Hymes, D. (1972), "Models of interaction in language and social life", en Gumperz and Hymes, D. (eds.), Directions in Sociolinguistics. The ethnography of communication, London, Basil.

Grimble, B. y Williams, T. (2004), "Student's perceptions of their information literacy skills in the media center", en Library media connection, 22(4), 26-28.

Instituto Nacional para la Evaluación de la Educación (2004), Resultados de las pruebas nacionales de aprovechamiento de lectura y matemáticas aplicadas el fin del ciclo 2001-2003, Joaquín Quesada García et al, México, D.F., Instituto Nacional para la Evaluación de la Educación.

, (2005) ¿Qué resultados obtuvieron las entidades en las pruebas nacionales de comprensión lectora y matemáticas? (Colección de folletos: los temas de la evaluación, 11) México, D.F., Instituto Nacional para la Evaluación de la Educación.

Kuhlthau, C. (1989), "Information search process: a summary of research and implications for school library media programs", en School Library Media Quarterly, 18, 19-25. , (1990), "The information search process: from the theory to practice", en Journal of Education for Library and Information Science, 31, Summer, 72-75. , (1991), "Inside the search process: Information seeking from the user's perspective", en Journal of the American Society for Information Science, Silver Spring, Md., American Society for Information Science and Technology, 42 (5), 361-371. , (2005), "Kuhlthau's information search process", en K. Fischer, S. Erdelez y L. MacKecnie, Theories of information behavior, Medford, N.J., Information Today, pp. 230-234.

Lebbin, V. (2005), "Students perceptions on the long-range value of information literacy instruction through a learning community", en Research strategies. 20 (3), 204-218.

Leontiev, A. N. (1993), Actividad, conciencia y personalidad, México, D.F., Asbe.

Mazón, N. (2006), Fortalecimiento del uso del habla exploratoria y la producción de macroestructuras de textos expositivos a través de una innovación educativa (Tesis doctoral, Universidad Nacional Autónoma de México, Facultad de Psicología, 2006).

Mazón, N., Rojas-Drummond, S., Vega G. y Vélez, M. (2006). “Colaboración, oralidad y alfabetización informativa: la creación de conferencias multimedia en alumnos de $6^{\circ}$ grado", en XIV Congreso Mexicano de Psicología, Puerto Vallarta, Jalisco. 
Mata, A. (2007), "Las prácticas de lectura entre estudiantes de secundaria que habitan en colonias marginadas de la zona norte de San Luis Potosí", en Memorias de las XXXVIII Jornadas Mexicanas de Biblioteconomía, México, AMBAC.

Newman, D., Griffin, P. y Cole, M. (1996), La zona de construcción del conocimiento : trabajando por un cambio cognitivo en educación, $2^{a}$ ed. Madrid, Morata.

Mercer, N. (1997), La construcción guiada del conocimiento, Barcelona, Paidós.

Nutefall, J. (2004), "Paper trail: one method of information literacy assessment”, en Research strategies, 20 (1-2), 89-98.

Meyer, B.J.F. (1984), “Texts dimensions and cognitive processing”, en H. Mandl, N. Steint, T. Eds., Learning and comprensión of texts, Hillsdale, N.J., Erlbaum.

Organización para la Cooperación y el Desarrollo Económico, (2001), Knowledge and skills for life : first results from PISA 2000, París, OCDE.

, (2003), PISA: Assesment framework: mathematics, reading, science and problem solving knowledge and skills, París, OCDE.

Palacios, C. y Vega G, (1994) Factibilidad de la educación de usuarios de la información en escuelas primarias del D.F., México, CUIB.

Poder Ejecutivo Federal. "Plan Nacional de Desarrollo 2007-2012 (2007)”, en Diario Oficial de la Federación, (31 de mayo del 2007), México, D. F., Gobierno de los Estados Unidos Mexicanos, Poder Ejecutivo Federal.

Ramírez, L., E. (2002), "La lectura: un problema para la sociedad de la información", en Investigación Bibliotecológica: archivonomia, bibliotecología e información, México, D.F., Centro Universitario de Investigaciones Bibliotecológicas, 16 (31).

, (2001), "La lectura en la sociedad contemporánea", en Investigación Bibliotecológica: archivonomia, bibliotecología e información, México, D.F., Centro Universitario de Investigaciones Bibliotecológicas, 15(30).

Rekrut, M. (1997), Colaborative research, en Journal of adolescent and adult literacy, 41 (1), 26-34.

Rodríguez G., A. (2007), "Definiendo la lectura, el alfabetismo y otros conceptos relacionados", en Investigación Bibliotecológica: archivonomía, bibliotecología e información, México, D.F., Centro Universitario de Investigaciones Bibliotecológicas, 21(42).

Rojas-Drummond, S.M., Albarrán, D. ; Vega, G. ; Zúñiga M. y Vélez, M. (2006), "Learning Togheter : the creation of communities of inquiry supported by ICT", en MirandaNet e-Journal. (http://www. mirandanet.ac.uk/internat/mexico.htm).

Rojas-Drummond, S.M., Mazón, N., Fernández, M. y Wegerif, R. (2006), "Explicit reasoning, creativity and co-construction in primary school children's collaborative activities", en Journal of Thinking Skills Creativity. 
Rojas-Drummond, S.M., Mercer, N. (2003), "Scaffolding the development of effective collaboration and learning", en International Journal of Education research, 39: 99-111.

Rojas-Drummond, S.M., Mercer, N. y Drabowski, E. (2001), "Collaboration, scaffolding and the promotion of problem solving strategies in Mexican pre-schoolers", en European Journal of Psychology of Education, 16 (2), 179-196.

Rojas-Drummond, S. M. y Peón, M. (2004), "Exploratory talk, argumentation and reasoning in mexican primary school children”, en Learning and instruction, 13 (86), 653-670.

Sánchez, E. (1993), Los textos expositivos, Madrid, Santillana.

Secretaría de Educación Pública, (2007), "Evaluación Nacional del Logro Académico en Centros Escolares”, en Sistema de Consulta de Resultados de la Prueba ENLACE, aplicada el pasado mes de junio del 2006 a más de 8.3 millones de niños y jóvenes de $3^{\circ}$ a $6^{\circ}$ grados de educación primaria y $3^{\circ}$ de educación secundaria, México, D.F., Secretaría de Educación Pública (http: www.snie.sep. gob.mx/enlace.asp; consultado: 8 de mayo del 2007). , (1993), Educación básica: primaria, plan y programas de estudio. México, D.F. : Secretaría de Educación Pública, Subsecretaría de Educación Básica y Normal, Dirección General de Materiales y Métodos Educativos.

Sine, L. (1994), "Teaching information skills at the primary grade levels", en School library media activities monthly, 10 (9), 29-30, 33.

Stalter, W.H., Graves, M.F. (1990), "Investigaciones sobre el texto expositivo: aportes para los docentes", en Texto expositivo: estrategias para su comprensión, Buenos Aires, AIQUE.

Street, Ch. (2005), "Tech talk for social studies teachers : evaluating online resources, the importancia of critical reading skills in online environments", en Social studies. 96(69), 272-273.

Stripling, B. y Pitts, J. (1988), Brainstorms and Blueprints: Teaching Research as a Thinking Process, Engelwood, CO, Libraries Unlimited.

Tastad, S.; Collins, N. (1997), "Teaching the information skills process and the writing process", en School library media quarterly, 25(3), 167-169.

Todd, R. (1995), "Information literacy: philosophy, principles, and practice”, en School libraries worldwide, 1(1), 54-68.

UNESCO (2005), Hacia las sociedades del conocimiento : informe mundial de la UNESCO, París, ONU, recuperado el 4 de mayo del 2007, en http://unesdoc.unesco.org/images/0014/001419/141908s.pdf

Utha State Office of Education (1991), Elementary and secondary core curriculum standards : levels K-1, Salt Lake City.

Vega, G., Rojas Drummond, S. y Mazón, N. (2007), "Diseño de programas de alfabetización informacional para educación primaria: una perspectiva sociocultural", en Memorias de las XXXVIII Jornadas Mexicanas de Biblioteconomía, 2 al 4 de mayo del 2004, León, Guanajuato, Asociación Mexicana de Biblioteconomía. 
Vega, G.; Rojas-Drummond, S., Rendón, J. y Mazón, N. (2004), “Cómo buscar, entender y presentar información ayudados por la tecnología: la enseñanza del texto expositivo en el programa 'Aprendiendo Juntos", en XII Congreso Mexicano de Psicología, Guanajuato, México, 22-24 de Septiembre.

Vygotsky, L.S (1979). El desarrollo de los procesos psicológicos superiores, Barcelona, Crítica.

Wang, P. y Soergel D. (1998), "A cognitive model of document use during a research project. Study I. Document selection”, en Journal of the American Society for Information Science, 49 (2), February.

Warmkessel, M. y McCade, J. (1997), "Integrating the information literacy into curriculum”, en Research strategies, 15(2), 80-88.

Wells, G. (2001), Indagación dialógica: hacia una teoría y una práctica socioculturales de la educación, Barcelona, Paidós.

Wenger. E. (2001/1998). Comunidades de práctica : aprendizaje, significado e identidad, Barcelona, Paidos.

Wilson, T. D. (2005), "Evolution in information behavior modeling: Wilson's model”, en E. Fisher, S. Erdelez y L. Mckechnnie (eds.), Theories of information behavior, Medford, New Jersey, Information Today.

Williams, D. y Coles, L. (2007), “Teacher's approaches to finding and using research evidence : an information literacy perspective", en Educational research, 49 (2), 185-206.

Winsconsin Education Media (1993), Information literacy : a position paper on information problem-solving, Madison, WEM. 